\title{
Marca-passo Permanente Após Implante Percutâneo Valvular Aórtico: a Necessidade é Maior que Imaginávamos?
}

\author{
Rogério Sarmento-Leite', Alexandre Schaan de Quadros ${ }^{1}$, Paulo Roberto Lunardi Prates ${ }^{1}$, Ismael Voltolini ${ }^{1}$, \\ Elias Conti ${ }^{1}$, Imarilde Giusti ${ }^{1}$, Paulo Affonso Salgado Filho' ${ }^{1}$, Gustavo Glotz de Lima ${ }^{1}$, \\ Carlos Antonio Mascia Gottschall ${ }^{1}$
}

\section{RESUMO}

Introdução: O implante percutâneo valvular aórtico é uma alternativa promissora no tratamento da estenose aórtica grave de pacientes com elevado risco cirúrgico. No entanto, parte desses pacientes pode desenvolver distúrbios do sistema de condução elétrico do coração e necessidade de implante de marca-passo permanente. O mecanismo das alterações do sistema de condução não está completamente elucidado. Nosso objetivo é avaliar a frequência e os fatores relacionados à necessidade de marca-passo permanente em nosso meio. Método: Série de casos com descrição das variáveis absolutas e relativas associadas à necessidade de marca-passo permanente em pacientes submetidos a implante percutâneo valvular aórtico no Instituto de Cardiologia do Rio Grande do Sul. Resultados: Entre novembro de 2008 e novembro de 2009, 10 pacientes foram submetidos a implante percutâneo valvular aórtico com prótese CoreValve ${ }^{\mathrm{TM}}$. Excluíram-se da análise 2 pacientes que faleceram por complicações não associadas aos distúrbios do sistema de condução. Nos 8 pacientes restantes obteve-se sucesso do procedimento, com redução dos gradientes entre o ventrículo esquerdo e a aorta e melhora sintomática. A maioria era composta por mulheres $(75 \%)$, com média de idade de 86 anos. Houve necessidade de marca-passo permanente em 6 pacientes (75\%) pós-implante percutâneo valvular aórtico. O único fator relacionado foi o surgimento de bloqueio de ramo esquerdo. No seguimento clínico, 1 paciente reassumiu o ritmo sinusal, 2 alternam ritmo próprio com o do marcapasso e 3 permanecem dependentes do estímulo artificial, até o momento. Conclusões: Observou-se elevada necessidade de marca-passo permanente após implante percutâneo valvular aórtico e o surgimento de bloqueio de ramo esquerdo parece estar associado a essa ocorrência.

\author{
ABSTRACT \\ Permanent Pacemaker After Percutaneous Aortic \\ Valve Implantation: Is the Need Greater than \\ We Had Anticipated?
}

Background: Percutaneous aortic valve implantation (PAVI) is a promising alternative treatment for severe aortic stenosis in high surgical risk patients. However, part of these patients may develop electrical conduction system disturbances and require permanent pacemaker implantation. The mechanism of such electrical conduction system disturbances has not been totally elucidated. Our objective is to assess the frequency and factors related to the need of a permanent pacemaker (PPM) in this scenario. Method: A series of cases describing absolute and relative variables associated to PPM implantation in patients submitted to PAVI at Instituto de Cardiologia do Rio Grande do Sul. Results: Between November 2008 and November 2009, ten patients were submitted to PAVI using the CoreValve ${ }^{\mathrm{TM}}$ prosthesis. Two patients who died due to complications not associated to the conduction system disturbances were excluded. The procedure was successfully carried out in the eight remaining patients, with gradient reduction between the left ventricle and aorta and symptomatic relief. Most of the patients were women $(75 \%)$ and mean age was 86 years. PPM was required in six patients $(75 \%)$ after PAVI. The single procedure related event was the development of left bundle branch block (LBBB). During clinical follow-up, one patient resumed sinus rhythm, two alternated their own rhythm with the pacemaker rhythm and three remain totally dependent of artificial stimulus. Conclusions: We observed an increased need of PPM after PAVI and the development of LBBB seems to be associated to it. Additional and powered studies,

\footnotetext{
1 Instituto de Cardiologia do Rio Grande do Sul/Fundação Universitária de Cardiologia - Porto Alegre, RS, Brasil.

Correspondência: Rogério Sarmento-Leite. Av. Princesa Isabel, 395 Setor de Hemodinâmica - Santana - Porto Alegre, RS, Brasil CEP 90620-001

E-mail: sarmentoleite@terra.com.br

Recebido em: 26/9/2009 • Aceito em: 1\%/12/2009
} 
Estudos adicionais com maior poder, comparativos com outras técnicas, são necessários para definir a exata incidência dos distúrbios de ritmo provocados pelo implante percutâneo valvular aórtico.

DESCRITORES: Estenose da valva aórtica. Valvas cardíacas. Implante de prótese de valva/métodos. Próteses valvulares cardíacas. Angiografia coronária.

$\mathbf{N}$ os últimos anos, o implante percutâneo valvular aórtico tem emergido como uma alternativa exequível e muito promissora no tratamento da estenose aórtica grave ${ }^{1-7}$. Essa nova abordagem terapêutica é destinada àqueles pacientes deferidos da abordagem cirúrgica clássica em função de elevado ou inaceitável risco de morbidade e mortalidade perioperatórias ${ }^{8}$.

Ao contrário do que ocorre na troca valvar aórtica cirúrgica, a área que circunda a válvula é coberta pela endoprótese, que comprime o ânulo valvular e estruturas próximas, incluindo os componentes do sistema de condução elétrica do coração. O nó atrioventricular e o ramo esquerdo do feixe de His trafegam no interior do corpo fibroso, adjacente à cúspide não-coronária da valva aórtica ${ }^{9,10}$, e podem ser afetados pelo dispositivo.

A incidência das alterações e o comprometimento do sistema de condução após troca valvar aórtica cirúrgica clássica são variáveis. Bloqueios de ramo esquerdo podem surgir em até 33\% dos casos e estar associados a pior prognóstico ${ }^{11,12}$. Já a necessidade de marca-passo permanente oscila entre $3 \%$ e $18 \%$, conforme a prótese utilizada ${ }^{12-18}$.

Nos casos de implante percutâneo valvular aórtico, esses achados ainda são muito heterogêneos e sua exata ocorrência é desconhecida, podendo estar sub ou superestimada. As séries publicadas apresentam resultados díspares e por vezes conflitantes, com números que variam de $6 \%$ a $33 \%^{19-22}$. No Brasil, a experiência clínica disponível na literatura ${ }^{23,24}$ com implante percutâneo valvular aórtico é incipiente e restrita ao uso do dispositivo CoreValve ${ }^{\mathrm{TM}}$ (Medtronic/ CoreValve, Inc. - Irvine, Estados Unidos).

A incidência e os fatores relacionados à necessidade de implante de marca-passo permanente ainda não foram avaliados em nosso meio e são o objetivo do presente estudo.

\section{MÉTODO}

Série retrospectiva de casos de implante percutâneo valvular aórtico por via retrógrada em pacientes com estenose aórtica grave e alto risco cirúrgico, realizados no Instituto de Cardiologia do Rio Grande do Sul (Por- comparing other techniques, are required to define the exact incidence of rhythm disturbances caused by PAVI.

DESCRIPTORS: Aortic valve stenosis. Heart valves. Heart valve prosthesis implantation/methods. Heart valve prosthesis. Coronary angiography.

to Alegre, RS) entre novembro de 2008 e novembro de 2009.

Excluíram-se pacientes com óbito hospitalar secundário a complicações não relacionadas ao sistema de condução.

Os critérios de indicação do procedimento foram a presença de estenose aórtica grave (área valvar $<0,8 \mathrm{~cm}^{2}$ ) em pacientes com idade $>80$ anos ou EuroSCORE logístico $>20 \%$ e com presença de dor torácica ou dispneia limitante, tonturas, pré-síncope, síncope ou arritmia ventricular.

Os casos foram avaliados por uma equipe multidisciplinar composta por um cardiologista clínico, um cardiologista intervencionista e um cirurgião cardíaco, sendo a troca valvular aórtica por cirurgia considerada de alto risco perioperatório.

A triagem dos pacientes envolveu a avaliação de condições anatômicas e funcionais favoráveis para o implante da prótese CoreValve ${ }^{T M}$. Para tanto, foram realizados ecocardiograma transtorácico com Doppler em cores, cineangiocoronariografia e angiotomografia de múltiplos detectores, conforme a necessidade.

A técnica de implante foi a padrão, supervisionada por um instrutor certificado e designado pela companhia responsável pela comercialização do dispositivo, mantendo a rotina do serviço, cuja experiência já foi preliminarmente descrita ${ }^{23}$. Foi utilizada a terceira geração da prótese CoreValve ${ }^{\mathrm{TM}}$, uma bioprótese de três folhetos de pericárdio porcino montados e suturados e um stent autoexpansível de nitinol (Figura 1), que posteriormente é ajustada a um cateter liberador, introduzido por uma bainha arterial $18 \mathrm{~F}$.

Em todos os pacientes foi realizado eletrocardiograma basal de 12 derivações, os quais receberam $500 \mathrm{mg}$ de ácido acetilsalicílico e 300 mg de clopidogrel na noite anterior à intervenção. Após a dose de ataque, a associação ácido acetilsalicílico (100 mg/dia) e clopidogrel $(75 \mathrm{mg} / \mathrm{dia})$ foi recomendada indefinidamente.

Os procedimentos foram realizados sob anestesia geral e em vigência de anticoagulação com heparina não-fracionada, ajustada pelo peso, para se atingir tempo de coagulação ativada de 300 a 350 segundos. 


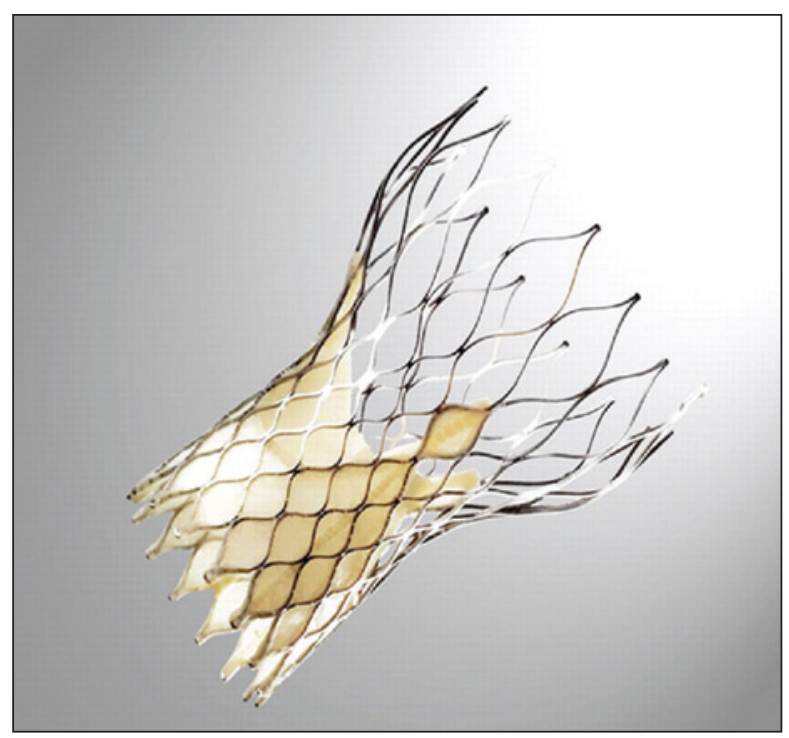

Figura 1 - Bioprótese CoreValve ${ }^{T M}$, composta por três folhetos de pericárdio porcino montados e suturados e um stent autoexpansível de nitinol.

Um eletrodo de marca-passo cardíaco temporário foi introduzido no início do procedimento pela veia jugular direita, para prevenção de bradiarritmias e para acelerar a frequência cardíaca (rapid pacing), no momento que precedeu a liberação da prótese.

Após o término dos procedimentos, os pacientes foram encaminhados para uma Unidade de Tratamento Intensivo (UTI), onde permaneceram por pelo menos 48 horas para observação e monitorização cardíaca contínua. Foram realizados eletrocardiogramas imediatamente após os procedimentos e diariamente até a alta hospitalar. O marca-passo temporário era mantido em regime de demanda e, quando necessário, conduzindo o ritmo cardíaco até a reversão da bradiarritmia ou o implante do marca-passo permanente. Considerou-se para implante de marca-passo permanente a presença de bloqueio atrioventricular total, com ou sem repercussão hemodinâmica, o aparecimento de bloqueio trifascicular ou fibrilação atrial com baixa resposta ventricular.

As seguintes variáveis foram avaliadas em relação à necessidade de marca-passo permanente:

- eletrocardiográficas: ritmo, frequência cardíaca, eixo elétrico, intervalo PR, duração e morfologia do QRS;

- angiográficas: calcificação do ânulo aórtico e profundidade do implante do dispositivo;

- ecocardiográficas: tamanho do anel valvar, espessura do septo interventricular no período diastólico final e fração de ejeção.

Os dados são apresentados de forma descritiva por meio de variáveis absolutas e/ou porcentuais, no intuito de identificar os fatores associados ao implante de marca-passo permanente.

O protocolo de pesquisa foi aprovado pelo Comitê de Ética em Pesquisa da Instituição e todos os pacientes forneceram termo de consentimento assinado. Este projeto não recebeu financiamento externo.

\section{RESULTADOS}

No total, 10 pacientes com diagnóstico de estenose aórtica grave sintomática e com risco operatório alto ou inaceitável foram selecionados para implante percutâneo valvular aórtico com o dispositivo CoreValve ${ }^{\text {TM }}$, dos quais 2 evoluíram para óbito por complicações clínicas ou do procedimento, não relacionadas ao sistema de condução. As características demográficas de base da população dos 8 pacientes em que foi obtido sucesso absoluto do procedimento, e que seguem em acompanhamento clínico, são descritas na Tabela 1.

Imediatamente após o procedimento, evidenciouse importante redução dos gradientes transvalvares aórticos médios, achado sustentado nas avaliações de curto (30 dias) e médio (6 meses) prazos (Figura 2).

À exceção de 3 pacientes readmitidos e posteriormente liberados no seguimento (um por insuficiência cardíaca descompensada por infecção respiratória, um por sangramento secundário a angiodisplasia de cólon e outro por infecção urinária), não foram verificadas outras intercorrências ou complicações, e todos se encontram bem, com melhora sintomática e estabilização clínica.

Do total de pacientes avaliados, 6 (75\%) necessitaram de marca-passo permanente (5 durante a internação e 1 no dia seguinte à alta hospitalar). A Tabela 2 descreve as razões para o implante de marca-passo permanente, o momento e o modo de estimulação utilizado.

A Tabela 3 mostra a análise comparativa dos achados eletrocardiográficos observados antes do implante percutâneo valvular aórtico, antes do implante do marcapasso permanente, e após 30 dias. O surgimento de bloqueio de ramo esquerdo foi o fator mais frequentemente associado ao desenvolvimento de bloqueio atrioventricular total na evolução e necessidade de marca-passo permanente.

A Tabela 4 exibe os achados ecocardiográficos (tamanho do anel valvar, espessura do septo interventricular e função ventricular) e angiográficos (grau de calcificação do ânulo aórtico e profundidade da prótese dentro do ventrículo esquerdo) dos pacientes que necessitaram implante de marca-passo permanente. Os 2 pacientes que não necessitaram marca-passo permanente em nossa série tiveram a prótese implantada com $\leq 10 \mathrm{~mm}$ de profundidade na cavidade ventricular esquerda. 
TABELA 1

Características de base da população

\begin{tabular}{|c|c|}
\hline Variáveis & $\mathbf{n}=\mathbf{8}$ \\
\hline Média de idade, anos & $86(80-94)$ \\
\hline Sexo feminino, n (\%) & $6(75)$ \\
\hline \multicolumn{2}{|l|}{ Sintomas, n (\%) } \\
\hline Dispneia & $8(100)$ \\
\hline Angina & $3(37,5)$ \\
\hline Síncope & $2(25)$ \\
\hline Tonturas & $3(37,5)$ \\
\hline \multicolumn{2}{|l|}{ Classe funcional de insuficiência cardíaca (NYHA), n (\%) } \\
\hline I & 0 \\
\hline$\|$ & $2(25)$ \\
\hline III & $5(62,5)$ \\
\hline IV & $1(12,5)$ \\
\hline Diabetes melito, n (\%) & $2(25)$ \\
\hline Hipertensão arterial, n (\%) & $5(62,5)$ \\
\hline Hipotireoidismo, n (\%) & $1(12,5)$ \\
\hline Cardiopatia isquêmica, n (\%) & $4(50)$ \\
\hline Cirurgia cardíaca prévia, n (\%) & $1(12,5)$ \\
\hline Angioplastia coronária prévia, n (\%) & $2(25)$ \\
\hline Insuficiência aórtica grave & 0 \\
\hline Creatinina basal média, mg/dl & $0,9(0,5-1,58)$ \\
\hline EuroSCORE logístico, \% & $23(8,97-34)$ \\
\hline Fração de ejeção do ventrículo esquerdo, \% & $71(64-75)$ \\
\hline Área valvar aórtica média, $\mathrm{cm}^{2}$ & $0,6(0,4-0,75)$ \\
\hline Gradiente transvalvar aórtico médio ao ecocardiograma, mmHg & $68(48-98)$ \\
\hline
\end{tabular}

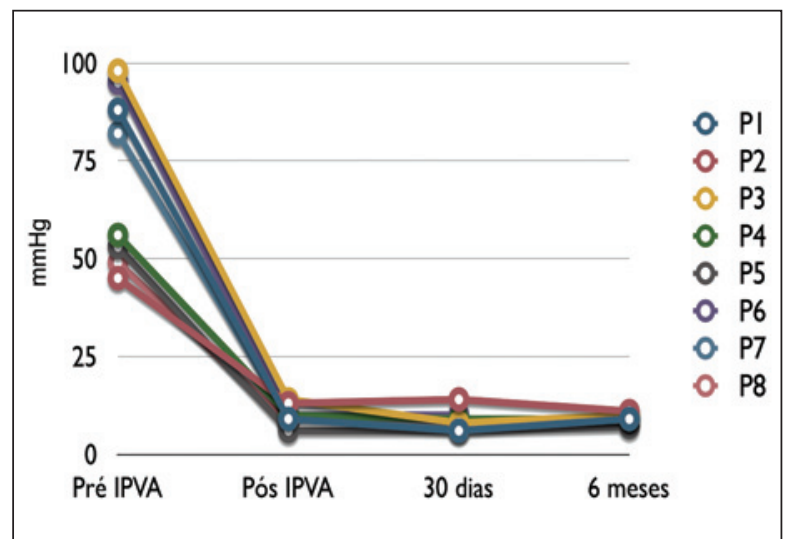

Figura 2 - Variação dos gradientes pressóricos médios medidos por ecocardiograma nos 8 pacientes analisados. Medidas realizadas antes e imediatamente após o implante percutâneo valvular aórtico, e 30 dias e 6 meses depois do procedimento. IPVA = implante percutâneo valvular aórtico; $\mathrm{P}=$ paciente.

Dos 6 pacientes que necessitaram de implante de marca-passo permanente, 1 reassumiu o ritmo sinusal (como ilustra a sequência da Figura 3), 2 alternam
TABELA 2

Motivo, momento do implante e modo de estimulação do marca-passo permanente após implante percutâneo valvular aórtico

\begin{tabular}{cccc}
\hline & Motivo & $\begin{array}{c}\text { Momento do } \\
\text { implante }\end{array}$ & $\begin{array}{c}\text { Modo de } \\
\text { estimulação }\end{array}$ \\
\hline MPP1 & BAVT & 8 dias & DDD \\
MPP2 & BAVT & 7 dias & DDD \\
MPP3 & BAVT & 2 dias & VVI \\
MPP4 & BAVT & 2 dias & VVI \\
MPP5 & BAVT & 1 dia & DDD \\
MPP6 & FA com $\downarrow$ RV & 2 dias & VVI \\
\hline
\end{tabular}

$\overline{\mathrm{BAVT}}=$ bloqueio atrioventricular total; FA com $\downarrow \mathrm{RV}=$ fibrilação atrial com baixa resposta ventricular; MPP $=$ marca-passo permanente.

ritmo próprio (sinusal em 1 paciente e fibrilação atrial em 1 paciente) com o de marca-passo, e 3 permanecem totalmente dependentes do estímulo artificial até o momento. 
TABELA 3

Análise comparativa dos achados eletrocardiográficos antes do implante percutâneo valvular aórtico, antes do implante do marca-passo permanente e após 30 dias

\begin{tabular}{|c|c|c|c|c|c|c|}
\hline \multirow[b]{2}{*}{ Paciente } & \multicolumn{2}{|c|}{ Índice } & \multicolumn{2}{|c|}{ Pré-MPP } & \multicolumn{2}{|c|}{30 dias } \\
\hline & Ritmo & BRE & Ritmo & BRE & Ritmo & BRE \\
\hline MPP1 & RS & Não & RS & Sim & RS & Não \\
\hline MPP2 & RS & Não & RS & Sim & MP & NA \\
\hline MPP3 & FA & Não & BAVT & NA & MP/próprio & Não \\
\hline MPP4 & RS & Não & BAVT & $\mathrm{NA}$ & MP/próprio & Não \\
\hline MPP5 & RS & Não & RS & Sim & MP & NA \\
\hline MPP6 & FA & Não & FA & Sim & MP & Não \\
\hline
\end{tabular}

$\mathrm{BAVT}=$ bloqueio atrioventricular total; $\mathrm{BRE}=$ bloqueio de ramo esquerdo; $\mathrm{FA}=$ fibrilação atrial; $\mathrm{MP}=$ marca-passo; $\mathrm{MPP}=$ marcapasso permanente; $\mathrm{NA}=$ não-avaliável; $\mathrm{RS}=$ ritmo sinusal.

TABELA 4

Achados ecocardiográficos e angiográficos dos pacientes que necessitaram marca-passo permanente

\begin{tabular}{|c|c|c|c|c|c|}
\hline \multirow[b]{2}{*}{ Paciente } & \multicolumn{3}{|c|}{ Ecocardiograma } & \multicolumn{2}{|c|}{ Angiografia } \\
\hline & $\begin{array}{l}\text { Anel } \\
\text { aórtico } \\
(\mathrm{mm})\end{array}$ & $\begin{array}{c}\text { Septo } \\
\text { interatrial } \\
(\mathrm{mm})\end{array}$ & $\begin{array}{l}\text { Fração de } \\
\text { ejeção } \\
(\%)\end{array}$ & $\begin{array}{c}\text { Calcificação } \\
\text { do ânulo aórtico } \\
(\mathrm{mm})\end{array}$ & $\begin{array}{c}\text { Profundidade da prótese } \\
\text { no ventrículo esquerdo } \\
(\mathrm{mm})\end{array}$ \\
\hline MPP1 & 27 & 10 & 74 & Moderada & 10,1 \\
\hline MPP2 & 30 & 11 & 72 & Moderada & 11 \\
\hline MPP3 & 33 & 11 & 61 & Moderada & 8,3 \\
\hline MPP4 & 26 & 13 & 76 & Moderada & 10,9 \\
\hline MPP5 & 34 & 14 & 64 & Moderada & 15,3 \\
\hline MPP6 & 28 & 10 & 75 & Moderada & 11,9 \\
\hline
\end{tabular}

MPP = marca-passo permanente.

\section{DISCUSSÃo}

Nossos achados demonstram elevada incidência de distúrbios avançados do sistema de condução com necessidade de implante de marca-passo permanente em pacientes submetidos a implante percutâneo valvular aórtico com a prótese CoreValve ${ }^{\mathrm{TM}}$, sendo um dos poucos estudos na literatura que tentam avaliar e identificar fatores associados a esse evento. Jilaihawi et al. ${ }^{19}$ reportaram incidência de marca-passo permanente de $33 \%$, superior aos $20 \%$ descritos por Piazza et al. ${ }^{20}$ ou os $7 \%$ descritos por Sinhal et al. ${ }^{21}$. Este último estudo, contudo, refere-se à análise da prótese de CribierEdwards $^{\mathrm{TM}}$ (Edwards Lifesciences - Irvine, Estados Unidos). O fato de essa prótese ser de aço inoxidável, montada com pericárdio bovino em stent expansível em balão e com comprimento menor, na comparação com a prótese CoreValve ${ }^{T M}$, sugere que algumas propriedade físicas e mecânicas das endopróteses podem influenciar diretamente a ocorrência dos distúrbios de ritmo $^{25}$. Nossa incidência de marca-passo permanente é superior à de todas essas séries, mas muito próxima aos $67 \%$ relatados por Brito Júnior et al. ${ }^{26}$, em outra experiência brasileira. Esses achados podem estar associados ao acaso ou ao fato de termos sido mais agressivos no manejo e na prevenção dos distúrbios de ritmo observados. Todavia, é inequívoco que a abordagem percutânea com a prótese CoreValve $\mathrm{T}^{\mathrm{TM}}$ parece determinar maior necessidade de marca-passo permanente, especialmente quando comparamos com o observado na troca valvar cirúrgica clássica $(3 \%$ a $18 \%)^{15-20}$ ou na valvuloplastia aórtica por balão paliativa $(4 \%)^{27}$.

Os achados de Jilaihawi et al. ${ }^{19}$ permitiram predizer com sensibilidade de $75 \%$, especificidade de $100 \%$ e acurácia superior a $90 \%$ os fatores que estiveram associados à necessidade de marca-passo permanente após implante percutâneo valvular aórtico com CoreValve ${ }^{\mathrm{TM}}$ : desvio do eixo elétrico para a esquerda no eletrocardiograma basal, surgimento de bloqueio de ramo esquerdo, e espessura do septo interventricular 
Sarmento-Leite R, et al. Marca-passo Permanente Após Implante Percutâneo Valvular Aórtico: a Necessidade é Maior que Imaginávamos? Rev Bras Cardiol Invas. 2009;17(4):476-83.

(> $17 \mathrm{~mm}$ ) e da cúspide não-coronária (> $8 \mathrm{~mm})$. Nossos achados são concordantes com o fato de o surgimento de bloqueio de ramo esquerdo ser um desses determinantes. Isso já havia sido observado por Piazza et al. ${ }^{20}$, que levantaram a hipótese de que o implante percutâneo valvular aórtico aumenta as chances de compressão e esmagamento do ramo esquerdo do feixe de His. Sugerem, também, que se existir protrusão demasiada da porção distal do dispositivo para o interior do ventrículo esquerdo, os fenômenos elétricos podem aumentar de forma expressiva, levando ao implante de marca-passo permanente. O presente estudo não tem poder para comprovar essa hipótese, embora a teoria seja plausível. Esse fato ( Figura 4) pode ser observado em um de nossos pacientes com implante de marca-passo permanente muito precoce, em decorrência de bloqueio atrioventricular de alto grau, intercalado com períodos de assistolia. A análise da angiografia e os ecocardiogramas realizados durante e após o procedimento são as formas mais práticas para aferir a posição final da prótese e especular sobre situações como esta.

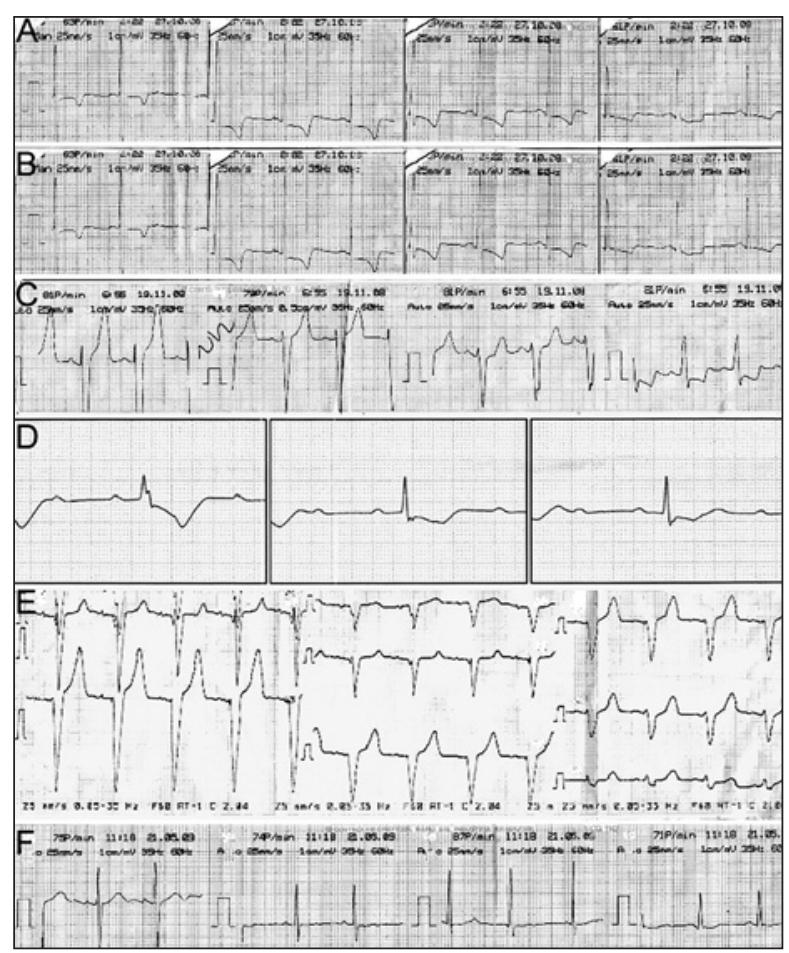

Figura 3 - Sequência dos eletrocardiogramas realizados na paciente MPP1. Em A, eletrocardiograma pré-procedimento, demonstrando ritmo sinusal. Em B, eletrocardiograma imediatamente pós-implante percutâneo valvular aórtico, superponível ao basal. Em C, eletrocardiograma realizado no sexto dia pós-implante percutâneo valvular aórtico, demonstrando bloqueio atrioventricular de primeiro grau e bloqueio de ramo esquerdo. Em D, eletrocardiograma realizado no oitavo dia, demonstrando bloqueio atrioventricular total. Em E, eletrocardiograma com ritmo de marca-passo. Em F, eletrocardiograma de controle 30 dias após implante percutâneo valvular aórtico, com retorno ao ritmo de base.
Não existe regra fixa para guiar o implante do marca-passo permanente nesse cenário. Até o momento, o implante deve ser embasado no julgamento clínico e na análise individualizada. A possibilidade de recuperação do ritmo sinusal e os riscos da manutenção de um eletrodo de marca-passo temporário por períodos prolongados (deslocamento com perda da condução, perfuração de ventrículo direito e infecção) devem ser sempre considerados. Por isso é necessária monitorização cardíaca contínua nos primeiros dias, realização de eletrocardiogramas rotineiros e atenção obsessiva para os distúrbios do ritmo e do sistema de condução.

Nossos achados não se prestam para inferências no que tange à influência da calcificação do anel aórtico e do tamanho do anel valvar na necessidade de marca-passo permanente. O primeiro, por ser de avaliação subjetiva, e o segundo, por ter apresentado grande variação nesta série. $O$ mesmo vale para função ventricular (normal em todos), espessura septal (distribuição homogênea em todos os pacientes), idade (octagenários ou nonagenários) ou gênero (maioria do sexo feminino).

Não interpretamos a necessidade de marca-passo permanente como complicação significativa do procedimento, mas como peculiaridade que requer atenta monitorização. É uma ferramenta adjunta que previne desfechos potencialmente adversos e pode impactar positivamente na qualidade de vida, especialmente naquelas populações mais idosas, com distúrbios de condução preexistentes ou disfunção autonômica. Abstraindo-se a questão custo, o implante de marcapasso permanente é um procedimento simples e com baixos índices de morbidade e mortalidade. Quando acertadamente proposto, melhora o débito cardíaco e a perfusão tanto cerebral como sistêmica, determinando maior bem-estar e podendo também prevenir morte súbita ou outras situações de risco. Nos pacientes que apresentam ritmo sinusal, apesar do bloqueio atrioventricular, devemos, sempre que possível, preferir o

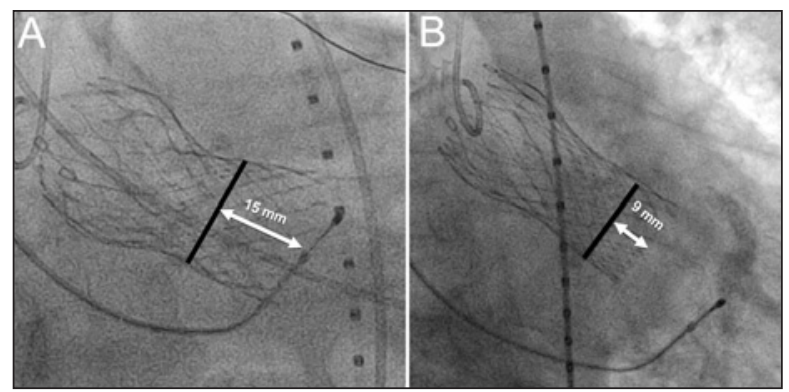

Figura 4 - Em A, imagem angiográfica pós-implante percutâneo valvular aórtico de paciente que necessitou de marca-passo permanente, demonstrando prótese com protrusão de $15 \mathrm{~mm}$ para o interior da cavidade ventricular esquerda. Em B, posicionamento ideal da prótese CoreValve ${ }^{T M}$. A distância entre a extremidade distal e o plano valvar é $<10 \mathrm{~mm}$. 
modo de estimulação DDD (dupla câmara), preservando o sincronismo atrioventricular e a colaboração atrial no débito cardíaco. O modo VVI deve ser restrito aos pacientes com fibrilação atrial permanente

O implante percutâneo valvular aórtico tem se mostrado capaz de reduzir os gradientes pressóricos, com melhora da qualidade de vida. Sabe-se que o sucesso do procedimento e sua eficácia dependem da adequada e firme expansão da prótese no ânulo aórtico, evitando ao máximo seu recolhimento elástico e vazamento perivalvular, que pode determinar insuficiência aórtica. Assim, torna-se inevitável a compressão do tecido fibroesquelético do coração e de seu sistema de condução, que trafegam adjacentes à cúspide nãocoronária da válvula aórtica ${ }^{9,10}$. Como o dispositivo em análise é montado em um sistema autoexpansível de nitinol, parece que a expansão não se encerra ao término do procedimento, determinando estresse mecânico, edema, reação inflamatória e isquemia no local da liberação. Imagina-se que esses distúrbios sejam passageiros, com retorno à condição basal ao longo do tempo. Isso, talvez, explique o fato de esses fenômenos de ritmo serem transitórios e de muitos pacientes readquirirem total ou parcialmente o ritmo basal com o tempo, conforme observado em alguns pacientes desta série. Isso também foi bem demonstrado por Jilaihawi et al. ${ }^{19}$, quando, após 30 dias, 3 dos 12 pacientes que receberam marca-passo permanente requeriam seu funcionamento por menos de $10 \%$ do tempo, enquanto nos restantes seu acionamento ocorria em $72 \%$ do tempo. O que ainda necessita ser elucidado é o exato comportamento temporal dessas ocorrências, permitindo que o paciente seja mais bem estratificado e manejado, fornecendo, assim, mais subsídios para a indicação precisa do implante de marca-passo permanente e do modo de estimulação elétrica.

\section{LIMITAÇÕES E IMPLICAÇÕES CLÍNICAS}

Este estudo, embora limitado por constituir-se em uma série de casos com amostra pequena, pela ausência de grupo controle e pelo seguimento tardio de todos os pacientes, alerta para uma ocorrência clínica que pode ser mais prevalente que imaginamos. Estudos adicionais com maior poder, comparativos com as abordagens terapêuticas tradicionais e de seguimento clínico mais prolongado, ainda são necessários para definir a prevalência e a incidência dos distúrbios de ritmo provocados por essa nova e promissora técnica. Nesse ínterim, considerando que os bloqueios sejam na porção intra ou infra His, o que pode tornar seu surgimento imprevisível, cabe o questionamento acerca da necessidade de implante de marca-passo permanente profilático para todos os pacientes submetidos a esse procedimento.

Nova geração de dispositivos de implante percutâneo valvular aórtico que permitam, sempre que necessário, seu reposicionamento durante a fase de liberação também é imperiosa, visto que isso pode interferir na necessidade de marca-passo permanente.

\section{CONCLUSÕES}

Nesta série de casos observou-se elevada necessidade de implante de marca-passo permanente após implante percutâneo valvular aórtico com prótese CoreValve ${ }^{\mathrm{TM}}$. O surgimento de bloqueio do ramo esquerdo pareceu estar associado a essa ocorrência.

\section{AGRADECIMENTOS}

Ao Dr. Eberhard Grube, pela orientação e colaboração na realização dos procedimentos; à empresa Endotec Brasil, distribuidora da prótese CoreValve ${ }^{\mathrm{TM}}$, pelo suporte logístico; e ao grupo médico, assistencial multiprofissional e administrativo do Instituto de Cardiologia do Rio Grande do Sul, pelo irrestrito apoio, que permitiu a operacionalização dos procedimentos, em especial a Daniel Ferreira, um de seus gerentes.

\section{CONFLITO DE INTERESSES}

Rogério Sarmento-Leite recebe apoio educacional das empresas Abbott, Biotronik, Cordis Co. e CoreValve; participa do Bureau de Palestrantes da Biotronik, B-Braun e CoreValve; e presta consultoria técnica às empresas Biotronik e Scitech. Alexandre Schaan de Quadros recebe auxílio de pesquisa das empresas Boston Scientific e Medtronic, Inc.; recebe apoio educacional das empresas Boston Scientific, Medtronic Inc., Cordis Co., B-Braun e Biosensors; e participa do Bureau de Palestrantes da CoreValve e Biosensors. Gustavo Glotz de Lima recebe apoio educacional do St Jude Medical; recebe auxílio de pesquisa das empresas Sanofi e Bristol Meyers Squibb; e participa do Bureau de Palestrantes da Libbs. Os demais autores declararam inexistência de conflito de interesses relacionado a este manuscrito.

\section{REFERÊNCIAS BIBLIOGRÁFICAS}

1. Cribier A, Eltchaninoff $H$, Tron C, Bauer F, Agatiello C, Sebagh L, et al. Early experience with percutaneous transcatheter implantation of heart valve prosthesis for the treatment of end-stage inoperable patients with calcific aortic stenosis. J Am Coll Cardiol. 2004;43(4):698-703.

2. Cribier A, Eltchaninoff $H$, Tron $C$, Bauer $F$, Agatiello C, Nercolini D, et al. Treatment of calcific aortic stenosis with the percutaneous heart valve: mid-term follow-up from the initial feasibility studies: the French experience. J Am Coll Cardiol. 2006;47(6):1214-23.

3. Grube E, Laborde JC, Gerckens U, Felderhoff T, Sauren B, Buellesfeld $\mathrm{L}$, et al. Percutaneous implantation of the CoreValve self-expanding valve prosthesis in high-risk patients with aortic valve disease: the Siegburg first-in-man study. Circulation. 2006;114(15):1616-24. Comment in: Circulation. 2007; 115(22):e612; author reply e613.

4. Grube E, Schuler G, Buellesfeld L, Gerckens U, Linke A, Wenaweser $\mathrm{P}$, et al. Percutaneous aortic valve replacement for severe aortic stenosis in high-risk patients using the 
second- and current third-generation self-expanding CoreValve prosthesis: device success and 30-day clinical outcome. J Am Coll Cardiol. 2007;50(1):69-76. Comment in: J Am Coll Cardiol. 2008;51(2):170; author reply 170-1.

5. Ye J, Cheung A, Lichtenstein SV, Pasupati S, Carere RG, Thompson CR, et al. Six-month outcome of transapical transcatheter aortic valve implantation in the initial seven patients. Eur J Cardiothorac Surg. 2007;31(1):16-21. Comment in: Eur J Cardiothorac Surg. 2007;31(1):7-8. Eur J Cardiothorac Surg. 2007;31(1):4-6. Eur J Cardiothorac Surg. 2007;32(1):188; author reply 188-90.

6. Lichtenstein SV, Cheung A, Ye J, Thompson CR, Carere RG, Pasupati $S$, et al. Transapical transcatheter aortic valve implantation in humans: initial clinical experience. Circulation. 2006;114(6):591-6. Comment in: Circulation. 2006;114(6): 533-5.

7. Webb JG, Pasupati S, Humphries K, Thompson C, Altwegg $\mathrm{L}$, Moss R, et al. Percutaneous transarterial aortic valve replacement in selected high-risk patients with aortic stenosis. Circulation. 2007;116(7):755-63.

8. Walther T, Falk V, Kempfert J, Borger MA, FassI J, Chu MW, et al. Transapical minimally invasive aortic valve implantation; the initial 50 patients. Eur J Cardiothorac Surg. 2008;33(6):9838. Comment in: Eur J Cardiothorac Surg. 2009;35(2):376-7; author reply 377-8.

9. Anderson RH, Ho SY. The anatomy of the atrioventricular node. Washington (DC): Heart Rhythm Society. [cited 2009 Jun 12]. Available from: http://hrsonline.org/uploadDocs/ AndersonHo_Anatomy_AVNode.pdf

10. Piazza $N$, de Jaegere P, Schultz C, Becker AE, Serruys PW, Anderson $\mathrm{RH}$. Anatomy of the aortic valvar complex and its implications for transcatheter implantation of the aortic valve. Circulation: Cardiovasc Intervent. 2008;1:74-81.

11. El-Khally Z, Thibault B, Staniloe C, Theroux P, Dubuc M, Roy D, et al. Prognostic significance of newly acquired bundle branch block after aortic valve replacement. Am J Cardiol. 2004;94(8):1008-11.

12. Thomas JL, Dickstein RA, Parker FB Jr, Potts JL, Poirier RA, Fruehan $\mathrm{CT}$, et al. Prognostic significance of the development of left bundle conduction defects following aortic valve replacement. J Thorac Cardiovasc Surg. 1982;84(3):382-6.

13. Limongelli G, Ducceschi V, D'Andrea A, Renzulli A, Sarubbi $B$, De Feo $M$, et al. Risk factors for pacemaker implantation following aortic valve replacement: a single centre experience. Heart. 2003;89(8):901-4.

14. Elahi M, Usmaan K. The bioprosthesis type and size influence the postoperative incidence of permanent pacemaker implantation in patients undergoing aortic valve surgery. J Interv Card Electrophysiol. 2006;15(2):113-8.

15. Dawkins S, Hobson AR, Kalra PR, Tang AT, Monro JL, Dawkins KD. Permanent pacemaker implantation after isolated aortic valve replacement: incidence, indications, and predictors. Ann Thorac Surg. 2008;85(1):108-12. Comment in: Ann Thorac Surg. 2008;86(4):1403.
16. Habicht JM, Scherr P, Zerkowski HR, Hoffmann A. Late conduction defects following aortic valve replacement. J Heart Valve Dis. 2000;9(5):629-32.

17. Kolh P, Lahaye L, Gerard P, Limet R. Aortic valve replacement in the octogenarians: perioperative outcome and clinical follow-up. Eur J Cardiothorac Surg. 1999;16(1):68-73.

18. Erdogan HB, Kayalar N, Ardal H, Omeroglu SN, Kirali K, Guler $M$, et al. Risk factors for requirement of permanent pacemaker implantation after aortic valve replacement. J Card Surg. 2006;21(3):211-5; discussion 216-7.

19. Jilaihawi $H$, Chin $D$, Vasa-Nicotera $M$, Jeilan $M$, Spyt $T, N g$ GA, et al. Predictors for permanent pacemaker requirement after transcatheter aortic valve implantation with the CoreValve bioprosthesis. Am Heart J. 2009;157(5):860-6.

20. Piazza N, Onuma Y, Jesserun E, Kint PP, Maugenest AM, Anderson $\mathrm{RH}$, et al. Early and persistent intraventricular conduction abnormalities and requirements for pacemaking after percutaneous replacement of the aortic valve. JACC Cardiovasc Interv. 2008;1(3):310-6.

21. Sinhal A, Altwegg L, Pasupati S, Humphries KH, Allard M, Martin $\mathrm{P}$, et al. Atrioventricular block after transcatheter balloon expandable aortic valve implantation. JACC Cardiovasc Interv. 2008;1(3):305-9.

22. Hernández-García JM, Muñoz-García AJ, Alonso-Briales JH, Jiménez-Navarro MF, Domínguez-Franco AJ, Rodríguez-Bailón I, et al. Experiência inicial em Málaga (Espanha) com prótese aórtica CoreValve para tratamento de estenose aórtica sintomática grave. Rev Bras Cardiol Invasiva. 2009;17(2):176-82.

23. Leite RS, Quadros AS, Prates PRL, Zanatta LG, Salgado Filho PA, Grando T, et al. Implante valvular aórtico percutâneo: experiência inicial do Sul do Brasil. Rev Bras Cardiol Invasiva. 2008;16(4):398-405.

24. Perin MA, Brito Júnior FS de, Almeida BO, Pereira MA, Abizaid A, Tarasoutchi $F$, et al. Substituição valvar aórtica percutânea para tratamento de estenose aórtica: experiência inicial no Brasil. Arq Bras Cardiol. 2009;93(3):299-306.

25. Masson JB, Kovac J, Schuler G, Yen J, Cheung A, Kapadia $S$, et al. Transcatheter aortic valve implantation: review of the nature, management, and avoidance of procedural complications. JACC Cardiovasc Interv. 2009;2(9):811-20.

26. Brito Júnior FS, Perin MA, Almeida BO, Pereira MAM, Abizaid A, Tarasoutchi F, et al. Substituição percutânea da valva aórtica com o sistema Corevalve para o tratamento da estenose aórtica, resultados do seguimento de médio prazo. Rev Bras Cardiol Invasiva. 2009;17(2 supl.1):11. Apresentado no 15응 Congresso da Sociedade Latino-Americana de Cardiologia Intervencionista; $31^{\circ}$ Congresso da Sociedade Brasileira de Hemodinâmica e Cardiologia Intervencionista; 14aㅡ Jornada Brasileira de Enfermagem em Hemodinâmica e Cardiologia Intervencionista. Rio de Janeiro, 10 a 12 junho 2009.

27. Percutaneous balloon aortic valvuloplasty. Acute and 30-day follow-up results in 674 patients from the NHLBI Balloon Valvuloplasty Registry. Circulation. 1991;84(6):2383-97. 